\title{
Postnatal Management Of Antenatally Detected Renal Pelvic Dilatation \\ Ahmed $\mathrm{M}$ and Alkhedir $\mathrm{A}$ \\ University Hospitals of Derby and Burton, United Kingdom \\ University Hospitals of Derby and Burton NHS Foundation Trust
}

\section{Background and Aims:}

Renal pelvic dilatation (RPD) is one of the most common abnormality detected on antenatal scans. RPD of $\geq 10 \mathrm{~mm}$ or bilateral RPD are perceived as significant abnormalities that warrant postnatal investigation. Similarly, presence of oligohydramnios and additional renal or extrarenal anomalies suggest significant pathology. Diverse practices exist on postnatal investigation and management of these children.

This study aimed to evaluate our current practice on postnatal management of RPD.

\section{Methods:}

Medical records of all infants who had renal pelvic dilatation detected on antenatal sonography between August 2015 and February 2018 were reviewed retrospectively to assess their postnatal management.

Results:

20 infants were found to have antenatal RPD with 35\% females and $65 \%$ males (Chart 1)). Only one fetus had bilateral RPD. With 9102 births in 31 months, RPD incidence was $0.22 \%$ (with $\geq 10 \mathrm{~mm}$ cut off at 34 weeks scan antenatally). All infants received prophylactic antibiotics prior to discharge after birth and had the first ultrasound renal scan as per protocol. 5 (25\%) infants had first postnatal scan with $<10 \mathrm{~mm}$ dilatation and were discharged. In 4 (20\%) infants, RPD resolved spontaneously within the next 12 months (Chart 2). 7 (35\%) were referred to urologist out of which $3(15 \%)$ required pyeloplasty (Box 1). One patient has persistent moderate RPD and continues to be followed up. 3 patients were either under or over investigated (Box 2). Only 1 patient developed coliform urinary tract infections.

\section{Conclusion:}

Majority of antenatally detected RPD resolved spontaneously with conservative management. $15 \%$ infants required surgical intervention. Role of prophylactic antibiotics requires further research.

\section{References:}

Am J Roentgenol. 1995 Mar;164(3):719-23

J Urol. 2017 Apr;197(4):1144-1149

J Urol. 2013 Aug;190(2):661-6

J Pediatr Urol. 2017 Jun;13(3):306-315

J Urol. 2017 Mar;197(3Pt2):831-837

J Urol. 2018 Jun;199(6):1615-1621

Arch Dis Child 2016;101:819-824
Chart 1: Male to female ratio $(N=20)$

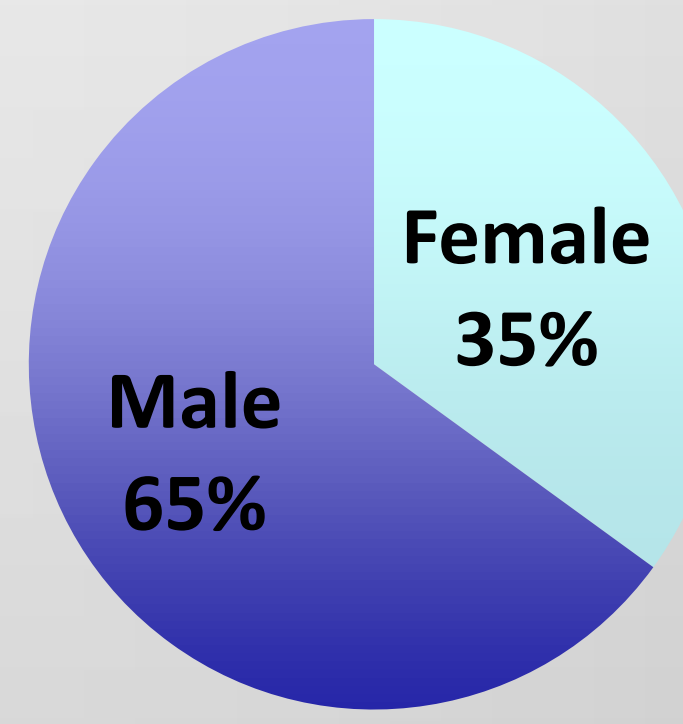

Chart 2: Optimum management $(\mathrm{N}=17)$

$$
\begin{aligned}
& 17 \text { had optimal management and } \\
& \text { follow-up }
\end{aligned}
$$

\section{$5(29 \%)$ had} RPD $<10 \mathrm{~mm}$

Discharged

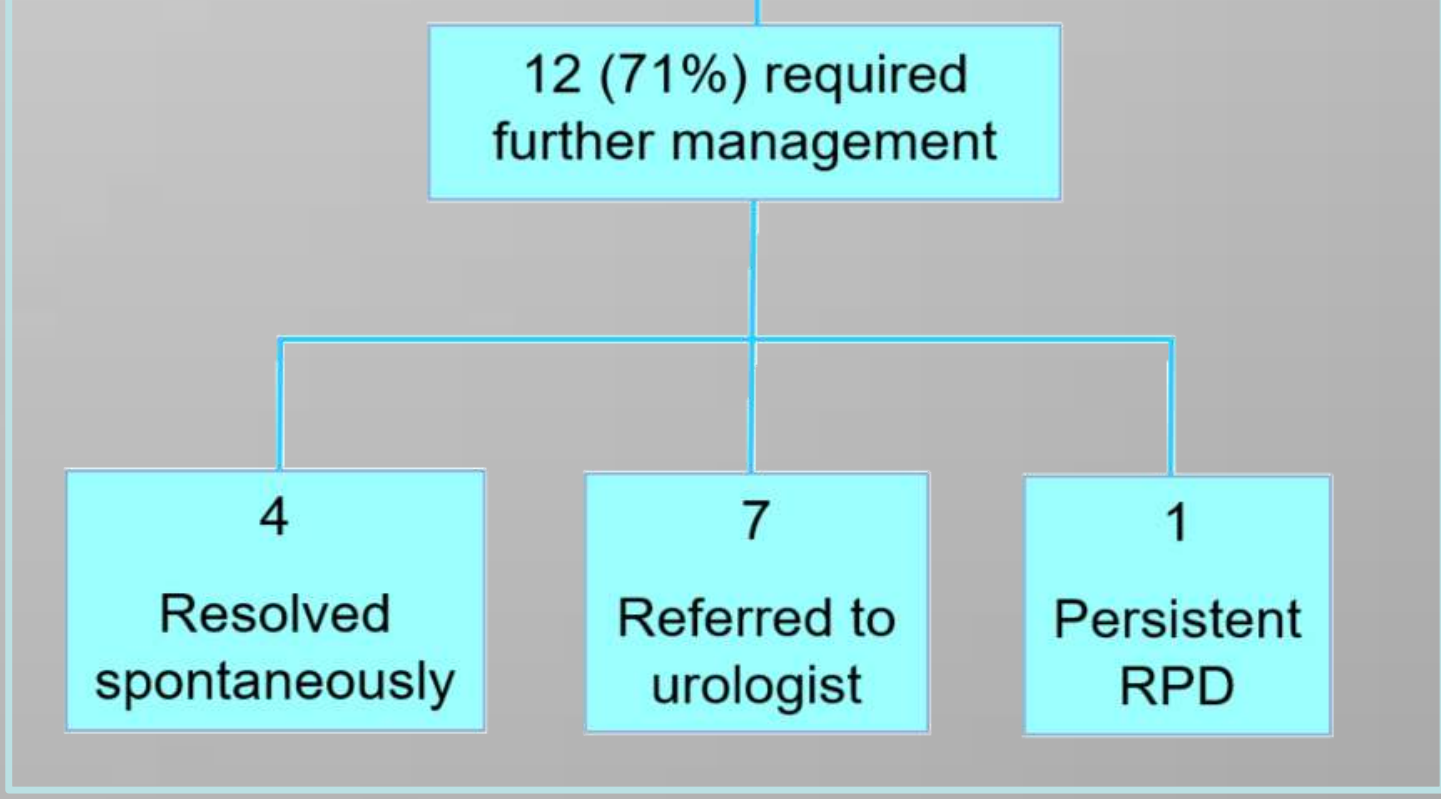

Box 1: Referral to urology $(\mathrm{N}=7)$

Reasons for referral

- PUJ obstruction (5)

- Ureteric dilatation (1)

- Significant RDP > 20 mm (1)

Of those who were referred to a urologist 3 out of $7(43 \%)$ required pyeloplasty.

One patient developed UTI.

Box 2: Non-adherence $(\mathrm{N}=3)$

Case 1

- Postnatal scan $<10 \mathrm{~mm}$ and continued prophylactic antibiotics and further renal scan arranged. Case 2

- Antenatal scan $<10 \mathrm{~mm}$ and prescribed prophylactic antibiotics and arranged postnatal renal scan.

Case 3

- Postnatal scan $10 \mathrm{~mm}$ and no further follow-up provided. 